\title{
A Conformal Preon Model
}

\author{
Risto Raitio \\ Espoo, Finland \\ Email: risto.raitio@gmail.com
}

How to cite this paper: Raitio, R. (2016) A Conformal Preon Model. Open Access Library Journal, 3: e3262. http://dx.doi.org/10.4236/oalib.1103262

Received: November 28, 2016 Accepted: December 17, 2016

Published: December 20, 2016

Copyright ( 2016 by author and Open Access Library Inc.

This work is licensed under the Creative Commons Attribution International License (CC BY 4.0).

http://creativecommons.org/licenses/by/4.0/

\begin{abstract}
I consider a preon model for quarks and leptons based on massless constituents having spin $1 / 2$ and charge $1 / 3$ or 0 . The color and weak interaction gauge structures can be deduced from the three preon states. Argument is given for unified field theory being based on gravitational and electromagnetic interactions only. Conformal symmetry is introduced in the action of gravity with the Weyl tensor. Electromagnetism is geometrized to conform with gravity. Baryon number non-conservation mechanism is obtained.
\end{abstract}

\section{Subject Areas}

Particle Physics

\section{Keywords}

Preons, Standard Model, Conformal Symmetry, Dark Energy, Dark Matter

\section{Introduction}

I reanalyze a model of quarks and leptons proposed in [1] [2] in order to find a guiding principle or cohesive symmetry to the model. The basic idea is to construct the quarks and leptons as three body bound states of two massless preons which have spin $1 / 2$, charge $1 / 3$ or 0 . The preons form a three-member combinatorial system for the $l=0$ standard model (SM) fermions. From this basis, the color and weak interactions of particles can be deduced. When the energy or temperature is above the quark and lepton bound state ionization value strong and weak interactions drop out. Unification of interactions was suggested on preon level in [2], with gravity and electromagnetism only contributing, but no unifying principle was introduced there. A method of unification by geometrization based on conformal symmetry is proposed in this note.

Conformal symmetry is introduced in the action using the Weyl tensor as described in [3] [4]. Gravity is quantized by coupling it to a quantized matter source [5] [6]. 
Electromagnetism is geometrized [7] to comport with gravity. A unitary, renormalizable quantum theory without ghosts is claimed. All symmetry breaking in the standard model is dynamical, quantum mechanical due to fermion bilinear condensates in the vacuum. The resulting mass scale produces curvature or Schwarzschild radius. In the absence of quantum mechanics the geometry is flat.

Why endorse preons? There are a few reasons: 1 ) they open the possibility to reduce the number of basic constituents, 2) help to understand, or deduce, the standard model non-Abelian gauge interactions, 3) massless preons are natural basis for conformal symmetry which facilitates geometrical unification of desired interactions, and 4) massless preons offer possibilities to construct conformal models of cosmology without dark matter or energy [8]. Unfortunately, testing this preon model can be done at present on Gedanken experiment level only.

This paper is organized as follows. In Section 2, I discuss the preon model in some detail. In Section 3, following Mannheim, a brief review is given of conformal gravity, its local gauge and conformal invariance, and geometrization of electromagnetism. Conclusions are given in Section 4.

\section{The Preon Model}

The preon model described in [2] is based on a statistical black hole model in loop quantum gravity (LQG). In LQG the geometry is quantized such that the lowest area eigenvalue is zero, which allows zero mass particles in the model construction as the Brown-York energy is proportional to area. The idea that a particle can be defined in pure gravity theory was first put forward in [9]. There it was also found that a charged black hole with Reissner-Nordström metric can have zero mass.

In the present note I start from a different basis, namely conformal symmetry. The virtue of conformal symmetry is that the action for conformal gravity is defined uniquely by the Weyl tensor, described in subsection 3.1. All particles in conformal theory are massless ${ }^{1}$ Therefore in this note I assume zero mass preons on action level.

Requiring charge quantization $\{0,1 / 3,2 / 3,1\}$ and preon permutation antisymmetry for identical preons, one can define three preon bound states which form the first generation quarks and leptons

$$
\begin{aligned}
& u_{k}=\epsilon_{i j k} m_{i}^{+} m_{j}^{+} m^{0} \\
& \bar{d}_{k}=\epsilon_{i j k} m^{+} m_{i}^{0} m_{j}^{0} \\
& e=\epsilon_{i j k} m_{i}^{-} m_{j}^{-} m_{k}^{-} \\
& \bar{v}=\epsilon_{i j k} \bar{m}_{i}^{0} \bar{m}_{j}^{0} \bar{m}_{k}^{0}
\end{aligned}
$$

The preons combine freely without other assumptions into standard model fermion bound states. They form a three member constrained combinatorial system. A binding interaction between preons is needed to make the quark and lepton bound states possible. I have at the moment no detailed form for this interaction. Its details are not expected to be of primary importance. I suppose this attractive, non-confining in${ }^{1}$ Conformal symmetry preserves angles, which guarantees the constancy of speed of light, i.e. the angle of light cone with respect to t-axis is 45 degrees. Mass can be generated to particles only dynamically. See subsection 3.1 . 
teraction is strong enough to keep together the charged preons but weak enough to liberate the preons at high temperature or after very long time period ${ }^{2}$. This interaction gives a small mass to quarks and leptons.

A useful feature in (2.1) with two identical preons ${ }^{3}$ is that the construction provides a three-valued subindex for quark SU(3) color, as it was originally discovered [10]. In addition, the weak SU(2) left handed doublets can be read from the first two and last two lines in (2.1). The SM gauge structure can be deduced in this sense from the present preon model. One could also have preon charges $1 / 3$ and $2 / 3$ but then the index $\mathrm{k}$ distinction between quarks and leptons in (2.1) would be lost.

One may now propose that, as far as there is an ultimate unified theory, it is a preon theory with only gravitational and electromagnetic interactions operating between preons. The strong and weak forces are generated in the early universe later when massless preons combine into quarks and leptons at lower temperature and they operate only with short range interaction within nuclei making atoms, molecules and chemistry possible. In a contracting phase of the universe processes take place in the opposite order.

The unification picture is supposed to hold in the present scheme up to the energy of about $10^{16} \mathrm{GeV}$. The electroweak interaction has the spontaneously broken symmetry phase below an energy of the order of $100 \mathrm{GeV}$ and symmetric phase above it. The electromagnetic and weak forces take separate ways at higher energies

(100 GeV «E $\ll 10^{16} \mathrm{GeV}$ ), the latter melts away due to ionization of quarks and leptons into preons, but the former stays strong towards Planck scale,

$M_{\mathrm{Pl}} \sim 1.22 \times 10^{19} \mathrm{GEV}$. Likewise the quark color interaction suffers the same destiny as the weak force. One is left with the electromagnetic and gravitational forces only at Planck scale.

Spin $3 / 2$ quarks and leptons are implied by this model. States with higher number of preons are possible but will not be considered here.

The proton, neutron, electron and $v$ can be constructed of 12 preons and 12 antipreons. The construction (2.1) is matter-antimatter symmetric on preon level, which is desirable for early cosmology. The model makes it possible to create from vacuum a universe with only matter: combine e.g. six $\mathrm{m}^{+}$, six $\mathrm{m}^{0}$ and their antiparticles to make the basic $\beta$-decay particles. Corresponding antiparticles may occur equally well.

The baryon number (B) is not conserved [11] [12] in this model: a proton may decay at Planck scale temperature by a preon rearrangement process into a positron and a pion. This is expected to be independent of the details of the preon interaction. Baryon number minus lepton number (B-L) is conserved.

\section{The Conformal Symmetry}

\subsection{Conformal Gravity}

Weyl introduced, while working on the geometrization of electromagnetism, a new

${ }^{2}$ Non-relativistically one may think of a potential wall or relativistically the SM vacuum energy is higher than the preon vacuum energy. As a first approximation this interaction can be introduced in flat spacetime.

${ }^{3} \mathrm{An}$ assumption appears here that the same charge preons inside quarks have the same spin $\mathrm{z}$-components. 
tensor called the Weyl or conformal tensor [13] (for an introduction to conformal theories, see e.g. [14])

$$
C_{\lambda \mu v \kappa}=R_{\lambda \mu \nu \kappa}-\frac{1}{2}\left(g_{\lambda v} R_{\mu \kappa}-g_{\lambda \kappa} R_{\mu \nu}-g_{\mu v} R_{\lambda \kappa}+g_{\mu \kappa} R_{\lambda v}\right)+\frac{1}{2} R_{\alpha}^{\alpha}\left(g_{\lambda v} g_{\mu \kappa}-g_{\lambda \kappa} g_{\mu v}\right)
$$

where $R_{\lambda \mu v \kappa}$ is the Riemann tensor. Under local conformal transformation $\omega(x)$ of the metric tensor $g_{\mu v}$ of the form

$$
g_{\mu \nu} \rightarrow \mathrm{e}^{-2 \omega(x)} g_{\mu \nu}
$$

the Weyl tensor transforms correspondingly as

$$
C_{\mu v \kappa}^{\lambda}(x) \rightarrow C_{\mu v \kappa}^{\lambda}(x)
$$

The pure local conformal gravity is based on the following action

$$
\begin{aligned}
I_{W} & =-\alpha_{g} \int \mathrm{d}^{4} x \sqrt{-g} C_{\lambda \mu \nu \kappa} C^{\lambda \mu \nu \kappa} \\
& =-2 \alpha_{g} \int \mathrm{d}^{4} x \sqrt{-g}\left[R_{\mu \kappa} R^{\mu \kappa}-\frac{1}{3}(R R)\right]
\end{aligned}
$$

where $\alpha_{g}$ is a dimensionless gravitational coupling constant. Conformal gravity is power-counting renormalizable and unitary [4]. In $I_{W}$ there is no cosmological constant term since $\int \mathrm{d}^{4} x \sqrt{-g} g_{\mu \nu} \Lambda$ is not conformal invariant. The cosmological constant will appear later when conformal symmetry is dynamically broken and gives the dimensionful $\bar{\psi} \psi$ a non-zero vacuum expectation value.

The reason for local conformal invariance is that massless particles move on the light cone which is invariant under the 15 parameter conformal group $S O(4,2)$ [6]. The covering group of $S O(4,2)$ is $S U(2,2)$. This is generated by the 15 Dirac matrices $\left(\gamma_{5}, \gamma_{\mu}, \gamma_{\mu} \gamma_{5},\left[\gamma_{\mu}, \gamma_{\nu}\right]\right)$ and its fundamental representation is a fermionic field. Therefore it is natural to take fermions as the basic objects in physics. Gravity is generated by gauging the conformal symmetry of the light cone. Mass scales are to be generated dynamically by fermion bilinear condensates in the vacuum.

Functional variation of $I_{W}$ with respect to metric defines a gravitational rank two tensor $W^{\mu \nu}$ in terms of the Riemann tensor that is covariantly conserved $\nabla_{\mu} W^{\mu v}=0$ and traceless $g_{\mu v} W^{\mu v}=0$. Assuming a conformally invariant matter action $I_{M}$ (an example is discussed in the next subsection 3.2 variation with respect to $I_{W}+I_{M}$ gives a fourth order derivative equation of motion of the form

$$
4 \alpha_{g} W^{\mu v}=T_{M}^{\mu v}
$$

By defining the left hand side of (3.5) as the energy-momentum tensor $T_{\text {gravity }}^{\mu v}$ we can write (3.5) in the form

$$
T_{\text {gravity }}^{\mu v}+T_{M}^{\mu \nu}=0
$$

We see that gravity and matter sectors are on equal footing and the total energymomentum tensor of the universe is zero.

The connection between Einstein gravity and conformal gravity is that the solutions of the former are solutions of the latter [15] [16]. However, conformal gravity has more 
general solutions to be explored. From these the Einstein solutions can be chosen by imposing a certain Neumann boundary condition on the metric at the future boundary. The boundary condition eliminates ghosts and the theory becomes an Einstein theory with a cosmological constant.

\subsection{Geometrization of Electromagnetism}

Conformal invariance is also important for understanding the geometrization of other interactions. Metrication of electromagnetism coupled to a Dirac field is given in [7].

The generalized Dirac action is

$$
I_{D}=\int \mathrm{d}^{4} x \sqrt{-g} i \bar{\psi} \gamma^{c} e_{c}^{\mu}(x)\left(\partial_{\mu}+\tilde{\Gamma}_{\mu}\right) \psi+h \cdot c .
$$

where $e_{c}^{\mu}(x)$ is a vierbein defined by $g_{\mu \nu}=e_{\mu}^{a} e_{\nu}^{b} \eta_{a b}$ (solving this for $\eta_{\mu \nu}$, the vierbein vectors are seen to diagonalize the metric tensor) and

$$
\tilde{\Gamma}_{\mu}(x)=\frac{1}{8}\left[\gamma_{a}, \gamma_{b}\right]\left(e_{v}^{b} \partial_{\mu} \mathrm{e}^{a v}+e_{\lambda}^{b} \tilde{\Gamma}_{v \mu}^{\lambda} \mathrm{e}^{a v}\right)
$$

which is obtained from the generalized connection

$$
\tilde{\Gamma}_{\mu \nu}^{\lambda}=\Lambda_{\mu \nu}^{\lambda}+W_{\mu v}^{\lambda}
$$

where $\Lambda_{\mu \nu}^{\lambda}$ and $W_{\mu \nu}^{\lambda}$ are the Levi-Civita and Weyl connections, respectively

$$
\begin{aligned}
\Lambda_{\mu \nu}^{\lambda} & =\frac{1}{2} g^{\lambda \alpha}\left(\partial_{\mu} g_{v \alpha}+\partial_{\nu} g_{\mu \alpha}-\partial_{\alpha} g_{\mu v}\right) \\
W_{\mu v}^{\lambda} & =-g^{\lambda \alpha}\left(g_{v \alpha} A_{\mu}+g_{\mu \alpha} A_{\nu}-g_{v \mu} A_{\alpha}\right)
\end{aligned}
$$

It turns out that the covariant derivative of the metric in (3.4) is non-zero, $\tilde{\nabla}_{\lambda}=-2 g^{\mu v} A_{\lambda}$, and therefore parallel transport is path dependent and the theory is untenable.

Secondly, in the Weyl connection drops out from the generalized Dirac action and therefore does not provide geometrization of electromagnetism. Replacing $W_{\mu \nu}^{\lambda}$ by

$$
\mathcal{W}_{\mu \nu}^{\lambda}=-\frac{2 i}{3} g^{\lambda \alpha}\left(g_{v \alpha} A_{\mu}+g_{\mu \alpha} A_{\nu}-g_{v \mu} A_{\alpha}\right)
$$

and $\partial_{\mu}$ by $\partial_{\mu}-2 A_{\mu}$ in (3.4) one ends up with a satisfactory action

$$
I_{D}=\int \mathrm{d}^{4} x \sqrt{-g} \mathrm{i} \bar{\psi} \gamma^{c} e_{c}^{\mu}(x)\left(\partial_{\mu}+\Gamma_{\mu}-i A_{\mu}\right) \psi
$$

The action $I_{D}$ is locally invariant under both gauge and conformal transformations $\omega(x)$

$$
\begin{aligned}
& \psi(x) \rightarrow \mathrm{e}^{-3 \omega(x) / 2} \psi(x) \\
& g_{\mu v} \rightarrow \mathrm{e}^{-2 \omega(x)} g_{\mu v} \\
& e_{\mu}^{\alpha} \rightarrow \mathrm{e}^{\omega(x)} e_{\mu}^{\alpha} \\
& A_{\mu}(x) \rightarrow A_{\mu}(x)
\end{aligned}
$$

One sees that $A_{\mu}(x)$ does not transform at all. The equation of motion for preons, without preon-preon interactions, is (3.6). 


\section{Conclusions}

The model proposed in this note makes an attempt to combine the phenomenological success of the standard model with one more fundamental layer of particles below the SM and apply the principle of conformal symmetry to the model. The preon layer allows one to deduce the non-Abelian interaction structure of the SM. Conformal symmetry is an important symmetry in gravity as well as in geometrization of other interactions, of which electromagnetism has a special role in the present scenario. The non-Abelian interactions of strong and weak forces are low energy short range interactions below the ionization energy of quarks and leptons. Open problems for future study include preon interactions and the three generations quarks and leptons. It has been my goal to explain the generations as preon level interactions but the fermion spectrum is too challenging. So far an elegant method for describing the three generations may be the framed standard model [17] [18].

In the models of quantum gravity mentioned above, LQG and conformal gravity, quantization takes place differently. LQG is a quantization approach for Hamiltonian formulation of pure Einstein gravity alone. The resulting geometry area and volume are quantized. Conformal gravity quantization is based on the quantized source of gravity. Electromagnetism can be metricated together with gravity. It remains to be seen which direction might be more fruitful in the future, both are geometric. Or will rather something else be needed.

To close this note, I mention that in the search of detailed models for the present scenario, it seems that large scale modeling in cosmology is close to the idea of conformal, or scale, symmetry as discussed extensively recently in [19]. Preons should be important in the very early and late phases of cosmological development. In the direction of Planck scale, or quantum gravity one may also have to consider models with deformation invariance more general than the conformal one [20] [21]. The meaning of preons is that they can give extra freedom in model building in various directions while keeping the number of basic interactions at two.

\section{References}

[1] Raitio, R. (1980) A Model of Lepton and Quark Structure. Physica Scripta, 22, 197. https://doi.org/10.1088/0031-8949/22/3/002

[2] Raitio, R. (2016) Combinatorial Preon Model for Matter and Unification. Open Access Library Journal, 3, e3032.

[3] Mannheim, P. (2016) Conformal Invariance and the Metrication of the Fundamental Forces. [arXiv: 1603.08405].

[4] Mannheim, P. (2016) Mass Generation, the Cosmological Constant Problem, Conformal Symmetry, and the Higgs Boson. [arXiv: 1610.08907].

[5] Mannheim, P. (2011) Intrinsically Quantum-Mechanical Gravity and the Cosmological Constant Problem. Modern Physics Letters A, 26, 2375. https://doi.org/10.1142/S0217732311036875

[6] Mannheim, P. (2012) Making the Case for Conformal Gravity. Foundations of Physics, 42, 388. https://doi.org/10.1007/s10701-011-9608-6 
[7] Mannheim, P. (2014) PT Symmetry, Conformal Symmetry, and the Metrication of Electromagnetism. [arXiv: 1407.1820].

[8] Mannheim, P. (2006) Alternatives to Dark Matter and Dark Energy. Progress in Particle and Nuclear Physics, 56, 340. https://doi.org/10.1016/j.ppnp.2005.08.001

[9] Einstein, A. and Rosen, N. (1935) The Particle Problem in the General Theory of Relativity. Physical Review, 48, 73. https://doi.org/10.1103/PhysRev.48.73

[10] Greenberg, O. (1964) The Color Charge Degree of Freedom in Particle Physics. [arXiv: 0805.0289].

[11] Bekenstein, J. (1972) Non Existence of Baryon Number for Static Black Holes I and II. Physical Review D, 5, 2403-2412. https://doi.org/10.1103/PhysRevD.5.2403

[12] Wheeler, J. (1971) Cortona Symposium on Weak Interactions. Edited by Radicati, L., Accademia Nazionale dei Lincei, Rome.

[13] Weyl, H. (1918) Sitzsungber. Preuss. Akad. Wiss, 465.

[14] Fabbri, L. (2008) Higher Order Theories of Gravitation. arXiv:0806.2610.

[15] Maldacena, J. (2011) Einstein Gravity from Conformal Gravity. arXiv:1105.5632.

[16] Anastasiou, G. and Olea, R. (2016) From Conformal to Einstein Gravity. Physical Review D, 94, Article ID: 086008. arXiv:1608.07826. https://doi.org/10.1103/physrevd.94.086008

[17] Chan, H.-M. and Tsou, S. (2015) The Framed Standard Model (I) and (II). arXiv:1505.05472, arXiv:1505.05473.

[18] Chan, H.-M. and Tsou, S. (1998) Physical Consequences of Non-Abelian Duality in the Standard Model. Physical Review D, 57, 2507-2522.

https://doi.org/10.1103/PhysRevD.57.2507

[19] Penrose, R. (2016) Fashion, Faith, and Fantasy in the New Physics of the Universe. Princeton University Press, Princeton. https://doi.org/10.1515/9781400880287

[20] Ivancevic, V. and Ivancevic, T. (2008) Undergraduate Lecture Notes in Topological Quantum Field Theory. arXiv:0810.0344.

[21] Teleman, C. (2016) Five Lectures on Topological Field Theory. Given at the CRM in Bellaterra, Barcelona. https://math.berkeley.edu/ teleman/math/barclect.pdf

\section{Submit or recommend next manuscript to OALib Journal and we will provide best service for you:}

- Publication frequency: Monthly

- 9 subject areas of science, technology and medicine

- Fair and rigorous peer-review system

- Fast publication process

- Article promotion in various social networking sites (LinkedIn, Facebook, Twitter, etc.)

- Maximum dissemination of your research work

Submit Your Paper Online: Click Here to Submit

Or Contact service@oalib.com 